\title{
Section 2: Solar Energy Flux Variations
}

\author{
H. S. Hudson \\ Space Sciences Laboratory, University of California, Berkeley
}

The chapters in this section of the monograph deal with the basic raw material of solar variability, namely the measurements themselves. With complete characterization of the spectral components of the irradiance, one might imagine an easy task in putting them all together to determine the energy flux from the Sun. As the length and depth of these chapters shows, however, the simple characterization of a spectral irradiance at the accuracies permitted by the available technology already becomes a sophisticated business. The scope of the problem becomes apparent when one realizes that the observed bolometric variability of the total irradiance does not exceed fractions of a percent, and has well-measured components with amplitudes below one part per million (Fröhlich)!

The introductory chapter of this session (Kuhn and Armstrong) deals with mechanisms; the subsequent chapters continue with reviews of the total-irradiance observations (Frölhich), the ultraviolet (Rottman, Floyd, and Viereck), the extreme ultraviolet (Woods and 13 co-authors). The knowledge thus obtained needs a synthesis for further research into causes and effects, and Thuillier et al. discuss the current best such synthesis.

Notably in this Section we find no chapters devoted exclusively to infrared and radio variability, an omission justified by the fact that these wavelength ranges are well-behaved and/or trivial as components of the total irradiance. Please see [Kundu et al., 1989] for further information on the longer wavelengths. We do find a chapter on solar energetic particles (Lario and Simnett); these also have no real significance in comparison with the total irradiance. But the particles may act as catalysts for important geophysical effects, as reviewed

Solar Variability and its Effect on the Earth's Atmosphere and Climate System

Geophysical Monograph

Copyright 2003 by the American Geophysical Union 
by Jackman and McPeters and Tinsley and Yu elsewhere in this monograph. Note also that the "luminosity" associated with the particles of the solar wind might approach one part per million of the total irradiance, not entirely negligible in view of the precision of present total-irradiance measurements now. Thus we must bear non-radiative components in mind as we discuss this subject. We might also note for completeness the neutrino energy loss from the solar core. This subject, including the extremely marginal evidence for variability, properly belongs again with the physicists now that the solar interior models have been vindicated [SNO Collaboration, 2002].

Many forms of solar irradiance variability - from the shortest time scales to the longest yet observable - results from solar magnetic activity, as discussed by Fox. Nevertheless we note that the power spectrum of total irradiance variability (see Fröhlich) contains both line and continuum components attributed to non-magnetic sources. The line features in the spectrum are the global oscillations described as p-modes in helioseismology, and also known as the 5minute oscillations. The spectral continua show us the solar surface effects of convection, specifically the granulation and supergranulation, as well as the irradiance anisotropies due to magnetic activity. To what extent does our knowledge of stellar convection permit us to understand the non-magnetic variations? Kuhn and Armstrong discuss some possible implications of our improving knowledge of convection, but observationally it remains unclear to what extent the simple picture derived from mixing-length theory may not suffice. We may be sure that, if non-mixing-length effects have not been observed yet, they will indeed be observed in the future. The measurement of solar irradiance variability (and related phenomena such as the shape of the Sun, as described by Kuhn and Armstrong), in fact provides an interesting channel for studies of the solar interior that is independent of helioseismology.

\section{REFERENCES}

Kundu, M. R., Woodgate, B. E., and Schmahl, E. J., Energetic phenomena on the sun, ASSL 153 (1989).

SNO Collaboration, Direct evidence for neutrino flavor transformation from neutral-current interactions in the Sudbury Neutrino Observatory, Phys. Rev. Lett. 89 (2002).

H. S. Hudson, Space Sciences Laboratory, University of California, Berkeley, CA 94720-7450, USA. (hhudson@ssl.berkeley.edu) 